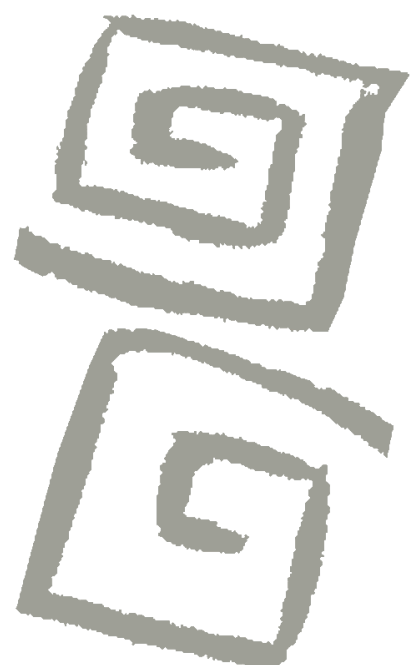

\title{
Un saber menos dado: nuevos posicionamientos en el campo de la salud mental colectiva
}

\author{
Less well-established forms of knowledge: new \\ positionings in the field of collective mental health
}

Angel Martínez-Hernáez ${ }^{1}$, Martín Correa-Urquiza² $^{2}$

${ }^{1}$ Doctor en Antropología Social. Profesor Titular, Departmento de Antropología, Filosofía y Trabajo Social; Medical Anthropology Research Center; Universitat Rovira i Virgili, Tarragona, España. $\bowtie$ iD

2 Doctor en Antropología Médica y Salud Internacional. Profesor Asociado, Departmento de Antropología, Filosofía y Trabajo Social; Medical Anthropology Research Center; Universitat Rovira i Virgili, Tarragona, España. $\triangle$ (iD)
RESUMEN La salud colectiva es ya un paradigma de largo recorrido en América Latina. La salud mental colectiva, por su parte, ha tenido un desarrollo relevante en algunos países latinoamericanos, e incluso ha actuado como estímulo para la reforma psiquiátrica en estos países. Sin embargo, ambas aparecen encapsuladas en un tiempo y territorio, entre otras cosas, por la hegemonía de una epistemología-mundo que, mientras ha impuesto un modelo naturalizado de verdad, ha negado la posibilidad de que otros saberes discutan sobre aquello ya ordenado (nosologías, protocolos, políticas, etc.), y menos aún sobre el propio ordenamiento. En este artículo reflexionamos sobre el poder de la etnografía, en tanto forma de conocimiento y relación social en sí misma, para ensanchar los espacios para una salud colectiva posible en un contexto donde aún es incipiente: Europa. La idea es que el punto de vista etnográfico facilita repensar lo ya dado, creando permeabilidad en las prácticas ya sedimentadas, así como nuevas ventanas de asombro. PALABRAS CLAVES Antropología Médica; Etnografía; Salud Colectiva; Epistemología; Europa.

\begin{abstract}
Collective health is a paradigm with a long history in Latin America. Similarly, collective mental health has had an interesting development in certain Latin American countries, even acting to stimulate psychiatric reform. However, both paradigms appear to be encapsulated in specific times and places, among other reasons because of a hegemonic global-scale epistemology that, by imposing a naturalized model of truth, denies other forms of knowledge the opportunity to question not only already-established disease categories, treatment protocols and health policies, but the established order itself. In this article, we reflect on the power of ethnography, as both a form of knowledge and a social relation in itself, to broaden the space available for a possible field of collective health in a context where it is still incipient: Europe. The ethnographic point of view allows us to rethink that which is already accepted, creating permeability in entrenched practices and opening up surprising new possibilities.
\end{abstract}

KEY WORDS Medical Anthropology; Ethnography; Collective Health; Epistemology; Europe. 


\section{INTRODUCCIÓN: DEL ASOMBRO}

"El conocimiento nace del asombro", decía Aristóteles, a lo que Canguilhem(1), en Lo normal y lo patológico, le añade su trazo particular al afirmar que el conocimiento propiamente vital es aquel que nace de la enfermedad. Ciertamente, el sufrimiento aparece como una encrucijada que mueve a la apertura de horizontes, al cuestionamiento y la desorientación. También a desaprender lo ya aprendido, tras percibir aquello que es fruto de sedimento como algo artificialmente naturalizado y que, podríamos añadir, ha constituido una imagen cómoda que no contesta nuestro mundo ni lo subvierte. Hay una incomodidad en el conocimiento -o quizá deberíamos decir, en el pensamiento, en tanto que su formulación es menos técnicaque nace de la interrogación y la reflexividad. Adicionalmente, hay un sufrimiento en la enfermedad y en la aflicción que paraliza el mundo vital, lo engulle y devuelve una consciencia lateral que permite pensar y pensarse a sí mismo de forma diferente. Cuando reivindicamos en el título de este artículo la expresión "un saber menos dado" apelamos a la relevancia de este tipo de mirada lateral para mantener el asombro. No nos referimos a la necesidad de una lateralidad vital ni tampoco ideológica, aunque podrían incluirse, sino al ejercicio de pensar y repensar desde otros itinerarios y aproximaciones lo ya pensado. Hablamos de ir al rescate del sujeto reflexivo que suspende el saber ya dado para explorar aquel que nace de la intersubjetividad y que permite construir lo colectivo.

Como es sabido, la salud colectiva es ya un paradigma de largo recorrido en América Latina y ha promovido mudanzas, no únicamente en las teorías y prácticas, sino también

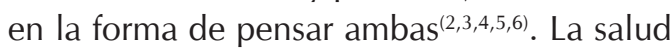
mental colectiva, por su parte, ha tenido un desarrollo relevante en algunos países latinoamericanos como Brasil y Argentina ${ }^{(7,8,9,10)}$, e incluso ha actuado como estímulo de una salud ampliada que observaba en la atención al sufrimiento psíquico una oportunidad de legitimación en un campo donde urgía la recuperación de lo colectivo para ofrecer un cauce a los procesos de reforma psiquiátrica. En Europa, sin embargo, tanto la salud colectiva como la salud mental colectiva son paradigmas con un reconocimiento aún limitado $^{(11)} y$, habitualmente, encapsulados en un tiempo y en un territorio, entre otras cosas por la hegemonía de una epistemologíamundo que, mientras ha impuesto un modelo geopolítico de verdad, evidencia y sentido, ha negado la posibilidad de que otros saberes y desde otros territorios se discuta sobre aquello ya ordenado (nosologías, protocolos clínicos, políticas, etc.) y, menos aún, sobre el propio ordenamiento.

La antropología médica o antropología de la salud ha aportado algunas claves para mantener el asombro y, en ese ejercicio, se ha aproximado a la tradición de la salud colectiva $^{(12,13)}$. Es por ello que no es inusual que entre estos campos disciplinarios exista un diálogo y una cierta reciprocidad que, por otro lado, apenas emerge en los núcleos hegemónicos de las ciencias de la salud en Europa. Una de estas claves aportadas tiene que ver con la resocialización y rehistorización de lo humano y, en el caso que aquí compete, del sufrimiento humano. Resocializar es mostrar la desigualdad social que subyace a los procesos de salud, enfermedad y atención, pero también destacar el papel de las hegemonías en la construcción de doctrinas generadoras de la ilusión de consenso cuando, en los hechos, impera el conflicto y la inequidad ${ }^{(14)}$. Hegemonías que facilitan las transacciones verticales hacia los colectivos subalternos desactivando la producción de sus propios saberes y también de sus contrahegemonías. Resocializar es, a la vez, retomar la enfermedad como una especie de "hecho biosocial total" en el que se condensa la complejidad de lo que somos, desde nuestra anatomía y fisiología hasta la corporalización (embodiment) de la economía política en esa misma anatomía y fisiología ${ }^{(14)}$.

Sin intención de ser exhaustivos, otra de las claves deviene, a nuestro juicio, del poder de la etnografía para sostener la incerteza como vía de conocimiento. La etnografía 
es un modo de pensar que, originalmente, responde más al objetivo antropológico de indagar y teorizar sobre cómo son las cosas -y sobre cómo han Ilegado a naturalizarse en una condición clausurada del ser-, que al propósito de prescribir cómo deberían ser. El objetivo etnográfico no se encuentra en la necesidad de dictaminar la normalidad o normatividad y sus opuestos (lo patológico, lo psicopatológico, lo deforme, lo grotesco, lo desviado, etc.), sino de comprender cómo son constituidos todos ellos en el juego social ${ }^{(15)}$. Elude, así, el posicionamiento sobre un saber ya dado para descubrir ese territorio que Weber definió como el "sentido de la acción social"(16) o Geertz como "el punto de vista nativo"(17). El trabajo etnográfico sitúa al investigador en la posición del no-saber, en una intersticialidad -que es también una variación de la lateralidad- semejante a la que Gadamer ${ }^{(18)}$ describió para el hermeneuta: un "estar entre" (Zwischen) un sentimiento de extrañeza o enajenación y un sentimiento de confianza o de pertenencia.

Se podrá objetar que el enfoque etnográfico no nace aparentemente con un propósito político ni de intervención, como es el caso de la salud colectiva y de la salud mental colectiva, sino de investigación. No obstante, y aun entendiendo que su nacimiento no tenga una funcionalidad política, es producto de un mundo sociopolítico, a la vez que puede devenir $-y$ ha devenido- en un instrumento político y de intervención. Pensemos en la antropología aplicada, que al indagar en el saber dado se crean también horizontes de transformación social. El marco epistémico de la etnografía supone un tipo de relación social que mueve a la intermediación y a habitar los intersticios sociales. El enfoque etnográfico es tanto un modo de conocimiento como una relación social en sí misma que ofrece un vínculo singular con los actores y colectivos sociales. Así, la acción del etnógrafo puede devenir en un hacer político que transforma las circunstancias en las que opera, ya que la investigación no puede desentenderse de aquello "en" y "con" lo que actúa, y que a menudo traspasa el propósito puramente analítico. La antropología médica se encuentra y funde en cierta manera con la salud colectiva y la salud mental colectiva desde el instante que se apropia de una voluntad transformadora, desde el momento que asume una cierta responsabilidad política con relación a aquello que analiza. A su vez, el posicionamiento etnográfico se puede convertir en la forma de conjurar el potencial efecto que se desprende de un "Yo ya sé cuáles son las necesidades de esta gente, de estos sujetos", cuando esas necesidades son pensadas desde la unilateralidad ideológica.

Cuando como investigadores asumimos que no sabemos, y no solo eso, cuando reconocemos un saber en los individuos y las poblaciones Ilamados profanos o legos, el conocimiento deviene un quehacer intersubjetivo. Lejos de la ilusión de lidiar exclusivamente con realidades fisiopatológicas, aquí se hace visible el sujeto que habita en el cuerpo, la voz del paciente, las narrativas de la aflicción. En un tiempo caracterizado por la preeminencia de la llamada "medicina basada en la evidencia" o "medicina basada en pruebas"(19) , quizá deberíamos recordar que lo que realmente es evidente en el campo de la atención en salud es el sufrimiento, y que dar cuenta de él o dialogar con él es también restituir la condición humana, social y subjetiva de la enfermedad, es ser más vínculo que certeza.

En el ámbito de la salud mental, ser vínculo es construir la problematización a partir de la demanda, del saber dado por otros sobre sus experiencias de aflicción. Es también recuperar el mundo social del sufrimiento, más allá de la sinécdoque organicista propia de la psiquiatría biomédica o de los excesos de un psicologismo en el que lo social o está ausente o es puramente fantasmático, pues se constituye como un "social interior" donde los sujetos emergen como islas psicopatológicas no afectadas por la marea de la vida social. Quizá sea debido a que gran parte de las disciplinas y aproximaciones teóricas en salud mental suelen aproximarse al sufrimiento a través de un marco de referencia que, mientras produce o reproduce las propias certezas, genera un encuadramiento unidimensional de lo que está 
en juego, generalmente circunscrito a lo que podemos Ilamar la demanda clínica. Y ahí otras demandas suelen desatenderse, como la demanda de equidad (vivienda, pensión, alimentación), la demanda política y ciudadana (la posibilidad de ser y estar siendo percibido desde otro lugar/identidad ligada a su condición de paciente o enfermo) o la demanda narrativa sobre el sufrimiento (lo que el "mundo hace con ellos" y no solo lo que "ellos hacen en el mundo"). Todas estas demandas desestimadas apelan, sin embargo, a instancias productoras de salud y de salud mental.

Tomando como punto de partida nuestra experiencia como investigadores, docentes y creadores de proyectos de innovación en antropología médica y salud mental colectiva, en este artículo queremos reflexionar sobre las aportaciones de la antropología médica $y$, especialmente, del punto de vista etnográfico para promover transformaciones sociales que permitan ensanchar los espacios para una salud mental colectiva posible. Con este propósito nos vamos a centrar en cuatros principios que consideramos fundamentales, aunque no constituyen un listado ni exhaustivo ni cerrado, pues el ánimo no es clausurar, sino mantener abiertas las ventanas que permiten el asombro.

\section{DE LA PERMEABILIDAD}

A nuestro juicio, pensar la salud mental colectiva implica asumir la necesidad de deconstruir ciertas rigideces de los saberes expertos y abrirnos a un cuestionamiento de lo que podríamos denominar como condición de impermeabilidad. Nos referimos a esa vocación de delimitar un ámbito científico propio, con sus taxonomías y parámetros actuando como límites que, a la vez, legitiman la existencia de la propia disciplina. Kleinman ${ }^{(20)}$ se ha referido a este tipo de ejercicio como la construcción burocrática del saber que, en este campo, podríamos añadir, se ha camuflado sobre constructos a menudo más retóricos que apegados a las prácticas reales. Un ejemplo de ello es definir el trastorno mental como un fenómeno biopsicosocial, mientras las prácticas muestran una jerarquía profesional y de legitimidades: lo bio como territorio del psiquiatra, lo psico como espacio del psicólogo, y lo social como arena del trabajador social ${ }^{(19)}$. No estamos negando aquí la necesidad de desarrollar un campo epistemológico que permita a cada disciplina habitar y habilitar una cierta geografía de prácticas, sino que cuestionamos el uso de los límites ficticios como muros defensivos que dificultan tanto el diálogo interdisciplinar como el abordaje de la salud. La sinécdoque organicista o psicologista es uno de los recursos de esta actitud defensiva, la impermeabilidad de los saberes uno de sus resultados.

Cada campo profesional implica un posicionamiento y un marco de referencia $y$, como tal, necesita de otros posicionamientos para encontrarse con un conocimiento atento a la complejidad de aquello que analiza. Asumir la incompletud del saber hace evidente la necesidad de una articulación permeable a la multiplicidad para consolidarse en su pretensión de abordar, explicar, tratar -en nuestro caso- la salud o la salud mental. Como indican Paim y Almeida Filho: "La salud colectiva no implica la adopción de un marco teórico de referencia exclusivo y excluyente" ${ }^{\prime \prime 21)}$, o cuando Liborio la define como un "conjunto articulado de prácticas técnicas, científicas, culturales, ideológicas, políticas y económicas, desarrolladas en el ámbito académico, en las instituciones de salud, en las organizaciones de la sociedad civil y en los institutos de investigación, informadas por distintas corrientes de pensamiento, resultantes de la adhesión o crítica a los diversos proyectos de reforma en salud"(22).

La salud colectiva es más bien un campo interdisciplinario de abordaje de los problemas estructurales e individuales relativos a la salud. De esta manera decimos que la salud mental colectiva puede pensarse desde ese esfuerzo por una permeabilidad que nos acerque a la construcción participativa, colectiva, de la aflicción. Hablamos de una 
porosidad o condición de permeabilidad asumible como posición dispuesta a la coalescencia, a fundirse "en" y "con" otros marcos de aproximación y referencia en favor de la generación de un contexto de posibles desde donde construir colectivamente los itinerarios para la recuperación. La salud mental colectiva emerge aquí como la necesidad de crear una estructura enunciativa, un marco de articulación sensible para sostener lo común en el campo de la salud. Hablamos de articular el abordaje desde un posicionamiento etnográfico atento al pluralismo epistémico que devuelva a los procesos de salud, enfermedad y atención su evidente multidimensionalidad.

Es palmario que aquella impermeabilidad de la que hablamos implica, a su vez, la constitución de relaciones de hegemonía/ subalternidad entre las mismas disciplinas y prácticas. Resulta obvio decir que, en los sistemas de salud contemporáneos, la preponderancia de la biomedicina coexiste con un posicionamiento subsidiario por parte del resto de disciplinas vinculadas al campo de la salud mental (sociales, psicológicas, sistémicas, etc.). Es decir, de muchas maneras y en muchas circunstancias estás últimas actúan como complemento que se suma a lo propuesto por el modelo médico hegemónico, en tanto punto de partida para pensar la realidad del fenómeno del sufrimiento. Dicho de otro modo: pueden discutir sobre lo ordenado, pero no sobre el ordenamiento. Pero es obvio, a su vez, que cada disciplina tiene la capacidad de aportar un marco de referencia propio, una manera de pensar la salud, la enfermedad y la atención que da sentido al proyecto de la salud colectiva y la salud mental colectiva.

Volviendo a la impermeabilidad, en el ámbito de la salud mental, dicha condición se manifiesta no solo en la relación entre aquellas disciplinas que intentan abordarla, sino también $y$, fundamentalmente, en el verticalismo inherente a la articulación de la terapéutica; en el monólogo de la aproximación biomédica ${ }^{(23)} \mathrm{o}$ en la constante condición de doble subalternidad, a la cual son relegados los sujetos de la aflicción, como saber no-experto y como sujeto de la norazón ${ }^{(24)}$. Esta circunstancia ha sido históricamente determinante en la articulación de las prácticas con relación a los saberes profanos o relativos a la subjetividad de las personas que viven procesos de sufrimiento psíquico. Diversos estudios etnográficos ${ }^{(25,26,27,28,29)}$ nos revelan que trabajar con narrativas nos abre un nuevo campo de conocimiento relativo a estas subjetividades. Nos ayudan a interpretar cómo es entendido el mundo desde el lugar de la aflicción. Se revelan saberes que existen y son activos y efectivos, a pesar de ser constantemente negados, ocultados, desatendidos. Saberes que, a su manera, cuestionan las lógicas biomédicas, y reconocen y defienden la existencia de otros fenómenos y otras prácticas que están más allá de la racionalidad instrumental en la construcción de la terapéutica y el conocimiento alrededor del sufrimiento psíquico. Estos son, a nuestro entender, los saberes profanos que se constituyen en su insistencia como la dimensión hereje -con relación a ciertos dogmas- que no ha dejado nunca de manifestarse a través de procesos que podemos pensar como de rebasamiento en relación con la posición de subalternidad que se le ha impuesto históricamente.

De esta manera, desde esta aproximación no son solo pertinentes las circunstancias objetivas de la enfermedad y del sufrimiento psíquico sino, al mismo tiempo, y sobre todo, la vivencia subjetiva de la aflicción y las estrategias que derivan de ella articuladas por los mismos individuos. En definitiva, qué es lo que los sujetos hacen con lo que les es dado en términos de sufrimiento: la "metabolización" subjetiva del proceso y sus reacciones. Partimos de la idea de que la enfermedad no es solo el cuerpo que evidencia un síntoma, un signo físico; no es algo que sucede exclusivamente en la dimensión somática. Nos interesan aquí las maneras a través de las cuales toda problemática incide sobre el mundo vital(30); cómo es vivido y actuado el proceso, y de qué manera afecta al entramado de la existencia. Siguiendo a Byron Good: la salud como la enfermedad no suceden en un cuerpo, sino en la vida ${ }^{(30)}$. 
La salud mental colectiva puede pensarse como un saber que se hace eco de una cierta condición de permeabilidad, de una predisposición a la coalescencia. Una condición que reside en la capacidad para asumir un cierto posicionamiento etnográfico, un estar de exégeta dispuesto a la decodificación de sentidos, a interpretar interpretaciones, a la pregunta y a la posibilidad de habilitar contextos para la reconstitución de una singularidad negada. En lo etnográfico reside, en parte, el mecanismo del encuentro con el otro, un lugar de incerteza asumida planteada como vía de conocimiento, un posicionamiento convocante de singularidad. La instancia de no-saber reconoce en su accionar una producción posible en el otro. Es por ello que la salud mental colectiva no es tanto un saber dado y específico, sino que se acerca más bien a un cierto tipo de posicionamiento epistemológico y político a partir del cual materializar el abordaje de la salud. Es una actitud, una disposición, que se reivindica en el pluralismo y en la coalescencia -más que complementariedad- de saberes y conocimientos articulada desde la horizontalidad. Es un marco de referencia que, en tanto suma posible de otros marcos, es un estado de alerta -y asombro-, una invitación al ejercicio hermenéutico como elemento inexorablemente ligado a la práctica cotidiana. Hablamos entonces de una inclinación a lo complejo. De aquello que reside en todo proceso de construcción compartida, de encuentro de saberes, de dialéctica no exenta de aquellos conflictos inherentes a la construcción de un saber en "equilibrio" que resulta del diálogo y la negociación permanente entre saberes individuales y colectivos.

En la salud mental colectiva hay poco de técnica adquirida, de protocolo pautado y establecido -eso quizás corresponda a cada una de las disciplinas y/o saberes que entran en juego en este ámbito-; hay, en cambio, una apertura a un cierto posicionamiento hacia lo permeable, hacia el encuentro, hacia lo que José Leal ha definido como saberes nómadas ${ }^{(31)}$. A nuestro entender la salud se crea en el proceso, se diseña y actúa en el encuentro. La salud mental colectiva es aquí definible a partir de una hermenéutica de las relaciones entre los diferentes saberes que operan en la construcción de la salud y, por lo tanto, es múltiple, mutable, dinámica y política.

\section{DE LA LATERALIDAD}

La lateralidad puede pensarse como un descentramiento de nuestra mirada que aporta nuevas perspectivas sobre lo observado. Nos aleja de lo predecible y en esa medida alimenta tanto el asombro como la creación de saberes nómadas. No se trata de la mirada lateral que imita otros saberes a los que desea emular, como nos indicó Canguilhem para las ideologías científicas ${ }^{(32)}$, sino más bien del salto del caballo que nos ilustra Canetti cuando dice:

No hay uniformidad en el verdadero saber. Todos los auténticos saltos se realizan lateralmente, como los saltos del caballo en el ajedrez. Lo que se desarrolla en línea recta y es predecible resulta irrelevante. Lo decisivo es el saber torcido, y sobre todo, lateral. ${ }^{(33)}$

La lateralidad implica asumir la permeabilidad y entender el marco de referencia sobre el que se construye y constituye cada disciplina, como un saber dado que precisa interaccionar con lo no dado, con lo que se produce en el vínculo con el otro. La lateralidad es encrucijada y se relaciona, así, con una predisposición a actuar por fuera de lo dado, una actitud de acogida hacia la posibilidad de desencriptar un conocimiento que no forma parte de nuestro marco de referencia. La lateralidad es, en parte, reconocer lo etnocéntrico en lo constitutivo de las prácticas y disciplinas para poder, si es el caso, neutralizarlo.

En el campo de la salud mental colectiva la lateralidad permite un pensar menos dado sobre las diferentes formas de sufrimiento psíquico, especialmente de la denominada locura y sus significados sedimentados. 
Pensemos que, en la historia de Europa, la locura ha venido asociada a una atribución de no-lugar social -que a su vez también es un lugar social-, ya fuese mediante la condena a un movimiento permanentemente desubicado, como El Quijote o la Stultifera Navis, o el confinamiento, como es el caso de la constitución del asilo como lugar de reclusión de no se sabe a ciencia cierta qué: ¿la sinrazón, el caos, la falta de sentido común, el desorden, el miedo, la diferencia, la disidencia?, pero que persiste durante siglos como una forma de control social(34). Movimiento y reclusión constituyeron dos prácticas sociales que conformaron un estar obs-ceno, en su sentido etimológico de fuera de escena, pues mientras el movimiento se convertía en una salida forzada o voluntaria del juego social, el confinamiento supuso una anulación de los derechos de ciudadanía.

Probablemente, esta atribuida obscenidad sea gregaria de la especial refracción de la locura con los procesos de hegemonía, persuasión e inculcación que operan habitualmente en la vida social y, en particular, con su opacidad, no a la razón, como generalmente se invoca, sino al sentido común entendido como sistema cultural, como conjunto de convenciones sociales que han sido naturalizadas hasta conformar nuestro paisaje cotidiano. La locura implosiona el sentido común, lo fragmenta y lo retuerce, aunque sea actuando al margen de las voluntades de quienes la sufren, para devolver una imagen desmoronada de él que podemos definir como sinsentido común o como sentido obs-ceno ${ }^{(34)}$. Esta refracción es la que induce a una gestión de la locura en los sistemas expertos que busca subsumir la vivencia de los afectados en categorías nosológicas previsibles, a la vez que disuade de cualquier mirada lateral en el campo profesional. Así, se facilita el énfasis del management frente a la narrativa, de la nosología frente a la experiencia, de la identificación burocrática sobre la identidad vivida: la ausencia de una mirada lateral es proporcional al miedo a la realidad psicótica y a las incertidumbres de su manejo.
Los conceptos de "identidad" e "identificación" son aquí utilizados como una variación y adaptación de las nociones desarrolladas por Terradas ${ }^{(35)}$ de identidad vivida e identificación jurídico-política. Según este autor, la identificación puede entenderse como una alienación de la vida, "la reducción y conversión abstracta de una memoria de vida en un signo de pertinencia o estigma político". En el ámbito que nos ocupa estaríamos oponiendo la identidad vivida a la identificación nosológica.

En este contexto, entendemos la lateralidad como un recurso para deshacer el nudo comunicativo que generalmente se instala en la visión por parte de los saberes expertos de fenómenos como la psicosis. Este nudo hace que la locura no se considere como necesaria de interpretación, de comprensión y, menos, de diálogo, quizá por considerarse como la simple expresión del error del juicio y del pensamiento, tal como indicaba tempranamente Kraepelinn ${ }^{(36)}$. Más bien, suele generar un monólogo en el que terapeutas, familiares y supuestos cuerdos acaban encontrando lo que buscaban: la sinrazón, la imposibilidad de un vínculo social del afectado o las propias nosologías como certezas que, a menudo, acaban generando una idea de enfermo total ${ }^{(25)}$ que requerirá de una terapia total ${ }^{(37)}$. La locura es algo que se da por sabido, aunque de ella no sepamos casi nada.

Sin lateralidad, sin descentrar nuestra mirada de los lugares comunes difícilmente podamos establecer un reconocimiento de la locura como realidad "con sentido" y también socialmente "consentida". En este punto podemos pensar en el legado de la antropología médica y el surgimiento de prácticas y experiencias concretas que fueron encontrando en la salud mental colectiva el espejo desde donde legitimarse, sostenerse y proyectarse. Experiencias como la de Radio Nikosia ${ }^{(25)}$, por ejemplo, fueron laboratorio de intenciones, una apertura, un cuestionamiento a la fosilización de la misma reforma psiquiátrica, un envite para pensar el sufrimiento mental más allá del encuadre biomédico, una intencionalidad política, una 
predisposición a la necesidad de articular una praxis de construcción compartida con los sujetos de la aflicción, de crear dispositivos para una arqueología de la subjetividad y la singularidad veladas. La lateralidad es, así, también el incentivo para una reforma psiquiátrica permanente.

\section{DE LA RECIPROCIDAD}

A pesar de que los sistemas expertos que trabajan habitualmente en salud mental han conformado un rico paisaje terminológico sobre lo social (psiquiatría social, salud mental comunitaria, atención psicosocial, salud mental colectiva, etc.), el ejercicio más común en las prácticas hegemónicas -especialmente de la psiquiatría biomédica-ha sido su desprendimiento. Un ejercicio que se expresa en la naturalización y encubrimiento de las relaciones sociales de producción, tanto de las enfermedades como de las formas de tratamiento y de terapia ${ }^{(37)}$. Así, en primera instancia el sujeto que sufre es transformado en objeto (la enfermedad, el trastorno, etc.) $y$, por lo tanto, reducido a una situación presocial, a un mundo de vísceras, disfunciones psicobiológicas y desequilibrios neuro-humorales y/o emocionales. En ello tiene que ver la aprehensión de los síntomas -en su definición de evidencia subjetiva de una enfermedad, aquello que dice el paciente- como si fuesen signos físicos, entendidos como evidencias objetivas de la enfermedad, la observación del clínico $^{(23)}$; de las narrativas como criterios diagnósticos, de la experiencia de sufrimiento como evidencia científica.

En segunda instancia, la desocialización del sujeto-paciente (ya reconvertido en nosología) evita cualquier interpelación, al menos en un sentido prioritario, sobre lo social del sujeto-profesional. De esta forma, las propias interpretaciones clínicas navegan autónomamente, libres ya de su contexto de producción y entendidas como meras copias de los hechos. Es el ideal baconiano del lenguaje científico como espejo de la naturaleza que deja de lado la biografía, las dificultades de ser-en-el-mundo, las adversidades y todo aquello que se resista a la objetivación. Los procesos de reificación adoptan, así, un carácter de doble negación de las relaciones sociales, tanto en el plano del sujeto-paciente como del sujeto-profesional. Si antes criticamos la percepción de las personas con sufrimiento psíquico como islas psicopatológicas, ahora podemos decir lo mismo de este ideal del sujeto-profesional ajeno a la marea del mundo, incluyendo sus propios prejuicios. Como decía Foucault ${ }^{(38)}$, la locura es en una sociedad; la terapia también, aunque se enmascare su dimensión social bajo un lenguaje técnico. Pero todo no queda aquí.

Mientras que en la imaginación biomédica el paisaje de los sujetos es cosificado, el de los objetos es a su vez personificado. Las enfermedades, los criterios diagnósticos, los trastornos, en tanto que categorías nosológicas, son los que cobran protagonismo e identidad en el lado del sujeto-paciente, mientras que en el del sujeto-profesional emergen la medicación, los tratamientos y las terapias, como entidades personificadas. Es, como decía Marx "el mundo encantado, invertido y puesto de cabeza en que Monsieur le Capital y Madame la Terre aparecen como personajes sociales, a la par que llevan a cabo sus brujerías como simples cosas materiales" ${ }^{\prime \prime 39}$. Ahora bien, lo que queremos destacar aquí es que el problema de la dialéctica de cosificación de los sujetos y personificación de las cosas es, de nuevo, la mediatización del encuentro intersubjetivo. ¿Qué tipo de encuentro implica la clínica, el que se establece entre dos sujetos o entre una nosología y un tratamiento habitualmente psicofarmacológico? ¿Hablamos de relaciones entre sujetos o entre los fantasmas o fetiches de las cosas?

En oposición a las relaciones de reciprocidad basadas en la tríada dar-recibir-retribuir y en las cuales el sujeto es orientado a un mundo externo en el que se produce un reconocimiento y subjetivización de un otro relacional (el pariente, el amigo, el vecino, etc.), en algunos tipos de relación clínica el sujeto es orientado a un mundo donde las cosas (trastornos, enfermedades, fármacos) 
son personificadas y los sujetos cosificados. De esta manera, la clínica se mimetiza con la relación consumidor-mercancía en la que el fetiche de la cosa -o quizá deberíamos decir de la mercancía- cobra vida. Y precisamente de esta falla surge el potencial de la reciprocidad como revulsivo.

Como cualquier relación social, la reciprocidad no es algo ajeno o externo al sujeto, sino una urdimbre que lo constituye, lo subjetiviza, lo "sujeta" a un mundo, pues le dota de entidad e identidad. La reciprocidad puede producirse entre iguales o entre individuos con posiciones asimétricas ${ }^{(40,41)}$, como aquellas que se establecen entre sistemas expertos y profanos; puede implicar ayuda mutua u otro tipo de transacciones; puede ser una reciprocidad generalizada o particular entre dos individuos; incluso puede hablarse de reciprocidad negativa $^{(42)}$, pero siempre reconoce a los sujetos de la relación, ya que habitualmente es más importante el sujeto-otro del vínculo (quién es) que la materialidad específica de la transacción (qué es). En esto se diferencia de otros tipos de relación sociales, como es la de consumidor-mercancía, en la cual lo que se prioriza es la materialidad de la transacción (la mercancía) frente a la identidad de los sujetos, que se desdibuja. En un campo en el que la identidad del paciente suele desdibujarse o redibujarse con base en artificios conceptuales, que en ocasiones son vividos como celdas, la reciprocidad da luz, "revela", en términos de Deligny ${ }^{(43)}$, y dota de existencia al otro. Lo hace partícipe del juego social. Lo constituye en su legitimidad de ser y estar en el vínculo.

La salud mental colectiva no puede pensarse con sujetos desdibujados, sin identidad vivida, capturados en una identificación ${ }^{(35)}$ burocrática del saber; sujetos sujetados a una trama relacional que les impide producir y reproducir su propia salud, a la vez que dialogar sobre su propio proceso de autorescate; sujetos, en fin, sin autonomía, sin reconocimiento, anclados a un ser sin historia; sujetos que podríamos definir como abiográficos, para los cuales, podemos pensar que la mejor terapia es, a menudo, tanto la narratividad, como reconocerse como partes activas de un vínculo. La relevancia de las relaciones de reciprocidad reside en que proporcionan un cobijo donde poder ser alguien.

\section{DE LA SINGULARIDAD}

En la atención en salud mental es notable el silenciamiento otorgado al decir y actuar de los sujetos de la aflicción con relación a su propio sufrimiento. En cierta forma, las personas han sido y son despojadas de la semántica que las nombra. Todo relato y constructo teórico relativo les es ajeno en su origen, pero se instala en ellas como "verdad total" que guarda rasgos de familia con la idea de institución total, pero también de enfermedad total y de terapia total de las que ya hemos hablado. Ese es el dominio simbólico del estigma. La colonización de la subjetividad se encarna y es al mismo tiempo en tanto colonización y cuestionamiento a una manera de percibir el mundo. La singularidad es capturada, asfixiada, domesticada a partir de un andamiaje taxonómico que suplanta toda reflexión subjetiva. Hay una cierta desnudez del "sí mismo" en los sujetos de la aflicción, una desnudez más ligada al despojo que a la des-estructuración propia de la denominada psicosis ${ }^{(24)}$. Más ligada a lo resultante de atravesar el bosque de símbolos que nombran el sufrimiento, que la realidad relativa al propio desencaje que significa la locura.

De diversas maneras, la identidad vivida es absorbida por la identificación clínica y fosilizada en un nombramiento dado. Las personas, circulen por donde circulen, se encuentran cercadas por la enunciación nosológica. No pueden ser más allá de aquello a partir de lo cual han sido nombradas. La identidad vivida se asfixia, o más bien, es asfixiada por la identificación patológica. Ante esto, es quizás necesario volver a decir que el problema no radica en la existencia del diagnóstico, sino en el traslado de ese diagnóstico como parte central de la identidad al campo de lo social, de la hegemonía de la identificación patológica sobre la identidad vivida. 
Ante esto, el posicionamiento etnográfico no implica partir de presupuestos sedimentados sobre el otro, sino que apunta a desentrañar las complejidades narrativas de lo subjetivo, se funda en la co-producción de lo singular, se abre a la consolidación de otras identidades potenciales. $Y$ aquí, lo singular hace referencia a lo que es propio, single, específico de cada sujeto. Lo que se constituye en el vaivén cotidiano entre psiquis y mundo y que deriva, ya no tanto de una imposición externa sobre el "deber ser", sino de decisiones y soluciones que surgen, en parte, de un cuestionamiento o un relativo estar "al margen" de los condicionantes sociales que materializan este tipo de opresión. Aquello que lo hace "sí mismo" y lo diferencia del resto mientras, al mismo tiempo, le permite sumarse a lo colectivo. Lo colectivo no puede pensarse sin lo singular. Desde allí, la salud mental colectiva puede pensarse en tanto generadora de un contexto potencial para lo singular. Un contexto que contribuya en la descolonización de los cuerpos, identidades y vidas, y también que ayude a desentrañar las condiciones de producción de esa colonización.

Cuando hablamos de dar lugar a lo singular, en nuestro caso, hacemos referencia no solo a la posibilidad de encuentro con los saberes profanos, sino a la necesidad de generar contextos desde donde el sujeto de la aflicción recupere una cierta autogestión de su mundo vital y de la semántica -tradicionalmente capturada por el relato biomédicoque construye su experiencia cotidiana; instancias e itinerarios que, al mismo tiempo, promuevan un "tramatizar lo disperso", como suele decir Olivera, fundador de Radio La Colifata ${ }^{(44)}$. Recordemos que lo singular tiene en el sufrimiento mental el obstáculo de la huella colonial sobre la subjetividad, sumado al obstáculo de la dispersión inherente en ocasiones a las características de la aflicción. El asombro hace lugar a lo singular. Devuelve la opción de su existencia, des-aprisiona.

\section{A MODO DE (IN)CONCLUSIÓN}

La salud mental colectiva atiende, quizás, a una cierta estructuralidad e historicidad en los determinantes de la aflicción y, al mismo tiempo, a la generación de contextos para la producción de singularidad. Puede pensarse como interfaz de encuentro permeable en pos de lo singular. Un lugar que aloje lo diverso y lo disperso, que sea, en tanto posibilidad abierta, aquel contexto de libertad donde los sujetos puedan reconstruir una semántica propia, nueva, desde donde pensar el mundo y pensarse en él. En definitiva, de la lateralidad del asombro en aquellos que se constituyen como interlocutores y de una cierta predisposición a la reciprocidad nace la posibilidad de lo permeable, del encuentro, de la coalescencia. Y, desde allí, deviene posible la generación de contextos para el rescate o la producción de la singularidad. La mirada etnográfica propia de la antropología y la antropología médica puede vislumbrarse como una herramienta para el encaje de la salud colectiva y la salud mental colectiva, especialmente en contextos nacionales, como es el caso de los europeos, donde estos paradigmas aún no han adquirido una posición de centralidad en las ciencias de la salud.

\section{AGRADECIMIENTOS}

Este artículo se ha desarrollado en el marco del proyecto "La vida medicada. Políticas sanitarias, consumo de antidepresivos y mundos vitales de los jóvenes catalanes financiado por el Plan Nacional de $\mathrm{I}+\mathrm{D}+\mathrm{i}$ del Ministerio de Economía y Competitividad de España (CSO2012-33841).

\section{REFERENCIAS BIBLIOGRÁFICAS}

1. Canguilhem G. Lo normal y lo patológico. México: Siglo XXI Editores; 1986.

2. Almeida-Filho N, Silva Paim J. La crisis de la salud pública y el movimiento de salud colectiva en Latinoamérica. Cuadernos Médico Sociales. 1999;75:5-30. 
3. Testa M. Pensar en salud. Buenos Aires: Lugar Editorial; 1993.

4. Breilh J. La determinación social de la salud como herramienta de transformación hacia una nueva salud pública (salud colectiva). Revista Facultad Nacional de Salud Pública. 2013;31(Suppl 1):13-27.

5. Granda E. ¿A qué llamamos salud colectiva, hoy? Revista Cubana de Salud Pública [Internet]. 2004;30(2) [citado 10 oct 2016]. Disponible en: http://tinyurl.com/k8azumj.

6. Spinelli H, (comp.). Salud colectiva: cultura, instituciones y subjetividad-epidemiología, gestión y políticas. Buenos Aires: Lugar Editorial; 2004.

7. Fagundes S. Águas da pedagogia da implicaçao: Intercessoes da educaçâo para políticas públicas de saúde. [Tesis de doctorado]. Porto Alegre: Universidade Federal do Rio Grande do Sul; 2006.

8. Ceccim RB. Invençâo da saúde coletiva e do controle social em saúde no Brasil: Nova educaçao na saúde e novos contornos e potencialidades à cidadania. Revista de Estudos Universitários. 2007;33(1):29-48.

9. Amarante P. Loucos pela vida: a trajetória da reforma psiquiátrica no Brasil. Rio de Janeiro: Editora Fiocruz; 2001.

10. Pitta AM. An assessment of Brazilian psychiatric reform: institutions, actors and policies. Ciência \& Saúde Coletiva. 2011;16(12):4579-4589.

11. Desviat M, Moreno A. De la psiquiatría comunitaria a la salud mental colectiva. En: Acciones de salud mental en la comunidad. Madrid: Asociación Española de Neuropsiquiatría (AEN); 2012. p. 126-136.

12. Nunes ED. A trajetória das ciências sociais em saúde na América Latina: revisão da produção científica. Revista de Saúde Pública. 2006;40:64-72.

13. Langdon EJ, Follér ML. Anthropology of health in Brazil: a border discourse. Medical Anthropology. 2012;31(1):4-28.

14. Martínez-Hernáez A. Mantener el asombro. Saúde \& Transformação Social / Health \& Social Change. 2011;2(1):i-iii.

15. Martínez-Hernáez A. La etnografía como dialógica: Hacia un modelo intercultural en las políticas sanitarias. En: Checa Olmos F, Arjona Garrido A, Checa Olmos JC, (eds.). Transitar por espacio comunes. Barcelona: Editorial Icaria; 2010. p. 57-83.
16. Weber M. Economía y sociedad. Madrid: Fondo de Cultura Económica; 1987.

17. Geertz C. "From the Native's Point of View": On the Nature of Anthropological Understanding. Bulletin of the American Academy of Arts and Sciences. 1974;28(1):26-45

18. Gadamer HG. Verdad y método. Salamanca: Sígueme; 1977.

19. Martínez-Hernáez A, Masana L, DiGiacomo SM, (eds.). Evidencias y narrativas en la atención sanitaria: Una perspectiva antropológica. Tarragona: Publicacions de la Universitat Rovira i Virgili; 2013.

20. Kleinman A. Rethinking Psychiatry: from cultural category to personal experience. New York: Free Press; 1988.

21. Silva Paim J, Almeida Filho N. A crise da Saúde Pública e a utopia da saúde coletiva. Salvador: Casa da Qualidade Editora; 2000.

22. Liborio MM. ¿Por qué hablar de salud colectiva? Revista Médica de Rosario. 2013;79:136-141.

23. Martínez-Hernáez A. What's behind the symptom?: On psychiatric observation and anthropological understanding. London: Taylor \& Francis; 2000.

24. Correa-Urquiza M. Radio Nikosia: la rebelión de los saberes profanos. Madrid: Grupo 5; 2015.

25. Corin EE. Facts and meaning in psychiatry: An anthropological approach to the lifeworld of schizophrenics. Culture, Medicine and Psychiatry. 1990;14(2):153-188.

26. Correa-Urquiza M, Silva T, Belloc M, Martínez Hernáez A. La evidencia social del sufrimiento: Salud mental, políticas globales y narrativas locales. Quaderns de I'Institut Català d'Antropologia. 2006;22:47-69.

27. Belloc M. Homem-sem-história: A narrativa como criaçao de cidadania. [Tesis de doctorado]. Tarragona: Universitat Rovira i Virgili; 2011.

28. Correa-Urquiza M. Radio Nikosia: Mutiny on the Ship of Fools. In: Araoz G, Alves G, Jawroski K. Rethinking madness: Interdisciplinary and multicultural reflections. Oxford: Interdisciplinary Press; 2013. p. 193-206.

29. Jenkins JH. Extraordinary conditions: Culture and experience in mental illness. Berkeley: University of California Press; 2015. 
30. Good B. Medicine, rationality and experience: An anthropological perspective. Cambridge: Cambridge University Press; 1994.

31. Leal J. Saberes nómadas: reflexiones sobre interdisciplinariedad en Salud Mental. X Jornada "Treball Social i Salut Mental en nens i adolescents: Mirades que sumen". Barcelona: FETB; 2013.

32. Canguilhem G. Idéologie et rationalité dans I'histoire des sciences de la vie. Paris: Librairie Philosophique J. Vrin; 1993.

33. Canetti E. El suplicio de las moscas. Madrid: Anaya \& Mario Muchnik; 1994.

34. Martínez Hernáez A. ¿Has visto como Ilora un cerezo? Pasos hacia una antropología de la esquizofrenia. Barcelona: Universitat de Barcelona; 1998.

35. Kraepelin E. Clinical Psychiatry: A Text book for students and physicians. New York: Macmillan Company; 1907.

36. Martínez-Hernáez A. Fuera de escena: la locura, lo obs-ceno y el sentido común. In: Martínez Hernaez A, Masana L, Digiacomo SM. Evidencias y narrativas en la atención sanitaria: Una perspectiva antropológica. Tarragona: Publicacions de la Universitat Rovira i Virgli; 2013. p. 199-220.
37. Foucault M. Obras esenciales. Barcelona: Paidós; 2013.

38. Marx K. Capital: A critique of political economy. Londres: Penguin Books; 1976.

39. Menéndez EL. El modelo médico hegemónico: transacciones y alternativas hacia una fundamentación teórica del modelo de autoatención en salud. Arxiu d'Etnografia de Catalunya. 1984;3:83-119.

40. Canals-Sala J. El regreso de la reciprocidad: Grupos de ayuda mutua y asociaciones de personas afectadas en la crisis del Estado del Bienestar. [Tesis de doctorado]. Tarragona: Universitat Rovira i Virgili; 2002.

41. Sahlins M. Economía de la Edad de Piedra Madrid: Akal; 1977.

42. Deligny F. Permitir, trazar, ver. Barcelona: Publicaciones MACBA; 2009.

43. Terradas I. La contradicción entre identidad vivida e identificación jurídico-política. Quaderns de I'Institut Català d'Antropologia. 2004;20:63-79.

44. Olivera A. La Colifata, relación entre Arte y Salud Mental. Clepios: Revista de Residentes de Salud Mental [Internet]. 2009;XV(1) [citado 10 oct 2016]. Disponible en: http://tinyurl.com/loc9sna. 